\title{
ON THE BIMODAL DISTRIBUTIONS OF HORIZONTAL BRANCHES
}

\author{
Young-Wook Lee, Pierre Demarque and Robert Zinn \\ Yale University Observatory
}

New synthetic horizontal branch (HB) models are presented for some globular clusters known to have bimodal HB distributions. These models are based on new Yale $\mathrm{HB}$ evolutionary tracks for $\mathrm{Y}=0.25$ and the core masses appropriate for the compositions. The distribution of stellar masses along the HB is given by a slightly modified version of Rood's (1973) function. Figure 1 compares the synthetic and the observed colormagnitude diagrams and the generalized histograms of the distribution of HB stars over (B-V)o (observational data from Alcaino \& Liller 1984, Buonanno et al. 1981, Stetson 1981, and Menzies 1974 for clusters M4, M5, N1851, and N6723 respectively). Following Norris (1981), we have used the period-luminosity-color relations to estimate the colors of some RR Lyrae variables. Since none of the parameters in the models has a bimodal distribution, the excellent agreement between the color distributions of the models and the observations suggests that the observed bimodal distributions are a consequence of the evolution from the zero-age HB. Contrary to the suggestion of Norris (1981) and Smith \& Norris (1983), there is no need to connect the bimodality of the HB with the observed bimodal $\mathrm{CN}$ distributions of the red giants in some of these clusters. The clusters in Figure 1 span a narrow range in metallicity $([\mathrm{Fe} / \mathrm{H}]=-1.40$ to -1.09 ; Zinn 1985$)$, and we are investigating other, less well observed, clusters in this range (e.g., N2808) to see if their bimodal $\mathrm{HB}$ distributions also have simple explanations.

This research was partially supported by NSF (AST-8304034) and NASA (NAGW-778) grants.

\section{REFERENCES}

Alcaino, G. and Liller, W. 1984 Astrophys. J. Supp1. 56, 19.

Buonanno, R., Corsi, C. and Fusi Pecci, F. 1981 Monthly Notices Roy. Astron. Soc. 196435.

Menzies, J. 1974 Monthly Notices Roy. Astron. Soc. 168, 177.

Norris, J. 1981 Astrophys. J. 248, 177.

Rood, R. 1973 Astrophys. J. 184, 815.

Smith, G. and Norris, J. 1983 Astrophys. J. 264, 215.

Stetson, P. 1981 Astron. J. 86, 687.

Zinn, R. 1985 Astrophys. J. 293, 424. 


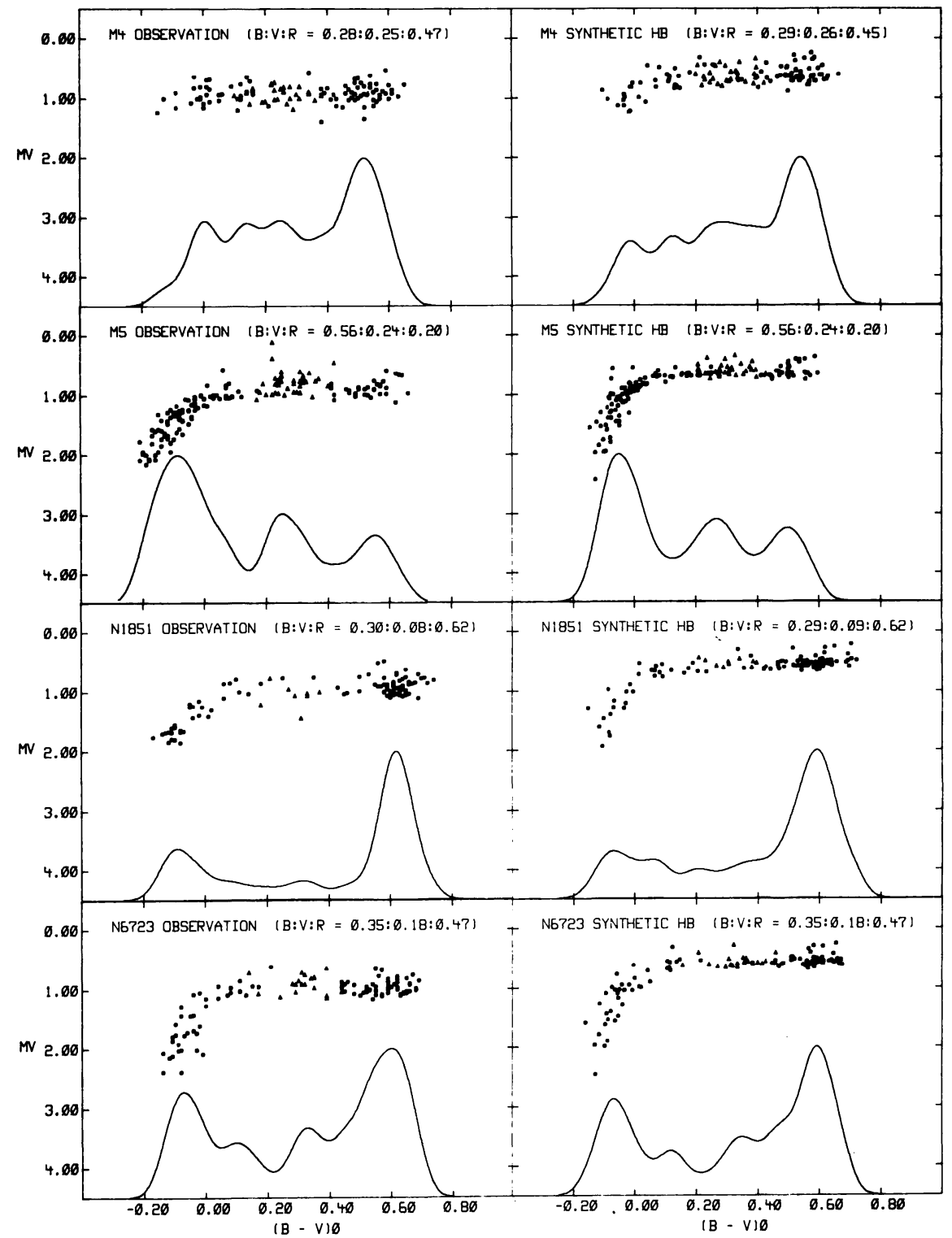

Fig. 1. Comparison of observations and synthetic HB's. Color-magnitude diagrams and generalized histograms (normalized to unity at the peak) are compared and proportions of BHB, RR Lyrae (triangles), and RHB stars (B:V:R) are indicated. The width of the variable strip is chosen to be 0.078 in $\log \left(\mathrm{T}_{\text {eff }}\right)$. The small differences in luminosity between the observations and the synthetic HB's are due to the choices for the distance moduli of the clusters and have no effect on the comparisons of the distributions in color. 